\title{
Inducible Yeast Two-Hybrid with Quantitative Measures
}

\author{
Jesus Hernandez*, Kevin D. Ross, and Bruce A. Hamilton \\ Department of Cellular and Molecular Medicine \\ Division of Medical Genetics \\ Institute for Genomic Medicine \\ Moores Cancer Center \\ UC San Diego \\ La Jolla, CA 92093-0644
}

*Present address: Sorrento Therapeutics, 4939 Directors Place, San Diego, CA 92121

\section{ORCIDs}

$\mathrm{JH}:$ 0000-0003-1548-0150

KDR: 0000-0003-1224-3950

BAH: 0000-0001-5599-9139 


\section{ABSTRACT}

The yeast two-hybrid ( $\mathrm{Y} 2 \mathrm{H})$ assay has long been used to identify new protein-protein interaction pairs and to compare relative interaction strengths. Traditional Y2H formats may be limited, however, by use of constitutive strong promoters if expressed proteins have toxic effects or post-transcriptional expression differences in yeast among a comparison group. As a step toward more quantitative $\mathrm{Y} 2 \mathrm{H}$ assays, we modified a common vector to use an inducible CUP1 promoter, which showed quantitative induction of several "bait" proteins with increasing copper concentration. Using mouse Nxf1 (homologous to yeast Mex67p) as a model bait, copper titration achieved levels that bracket levels obtained with the constitutive $A D H 1$ promoter. Using a liquid growth assay for an auxotrophic reporter in multiwell plates allowed log-phase growth rate to be used as a measure of interaction strength. These data demonstrate the potential for quantitative comparisons of protein-protein interactions using the $\mathrm{Y} 2 \mathrm{H}$ system. 


\section{INTRODUCTION}

Identifying quantitative changes in protein-protein interaction (PPI) networks caused by allelic variation in a protein of interest remains technically challenging. This is especially true for natural variation consistent with grossly normal protein function, where the expectation might be difference in relative strength of interactions rather than qualitative differences in number or identity of interactions. Potential for covariation among protein sequence, abundance, and conformational states further complicates most simple assays. An additional complexity for cellular assays, which may provide needed context for some interactions, is that high expression of the tested protein interfaces may by itself pose toxicity, complicating the interpretation of terminal readout measures. The mRNA nuclear export factor NXF1 may serve as an example: homologous to yeast MEX67p, it is an essential gene with a highly conserved core PPI network [1-11], yet a single amino acid substitution in mice $(E 610 G)$ creates a potent genetic suppressor of intronic retroviral insertion mutations without changing apparent steady-state NXF1 protein levels [12-14].

One approach to identifying quantitative differences in interaction strength is to test the strength of reporter expression in a yeast two-hybrid $(\mathrm{Y} 2 \mathrm{H})$ assay $[15,16]$. This easilymodified approach has several variants that have in common use of a hybrid protein with a sequence-specific DNA binding domain (e.g., GAL4-DBD) fused to a protein of interest (the "bait") and a second hybrid protein with a transcriptional activation domain fused to a potential bait-interacting protein (the "prey"). Physical interaction between bait and prey in the nucleus constitutes a bipartite transcription factor that drives expression of one or more reporter genes in proportion to the level of expression and strength of interaction between the bait and prey. Prior work has used both colonial growth on solid media and liquid growth combined with auxotrophic, enzymatic, or fluorescent reporters [15-21], but typically do not allow adjustment for differences in expressed protein levels. 
Using a strong constitutive promoter to drive high-level expression generally provides good sensitivity for interactions, but can be a limitation in some applications. The classic GAL4-based Y2H plasmids, for example, use a constitutive ADH1 promoter. In studies preliminary to the work presented here, application of this system to allelic Nxf1 bait proteins from divergent mouse strains, C57BL/6J (B6) and CAST/EiJ (CAST), achieved relatively low and unequal expression levels from the $A D H 1$ promoter in yeast, potentially by competing with endogenous Mex67p for a more limiting factor. To address this, we modified the pGBKT7 bait vector to include a synthetic version of the copperinducible CUP1 promoter, which can be quantitatively induced by addition of copper ions to the media [22-26]. The modified vector allowed titratable expression of multiple fusion proteins and allowed functional titration of $\mathrm{Y} 2 \mathrm{H}$ interaction using growth rate in liquid culture as an assay for an auxotrophic reporter. Varying copper ion concentration from 0 to $200 \mu \mathrm{M}$ permitted a range of expression levels that bracketed the level achieved with the $A D H 1$ vector. Inducible $\mathrm{Y} 2 \mathrm{H}$ should have applications for bait proteins that are mildly toxic under constitutive conditions or for quantitative comparisons of affinity between bait proteins with different steady-state levels in yeast, by allowing for interaction measures across expression levels.

\section{MATERIALS AND METHODS}

Plasmid construction. Yeast Matchmaker Two-Hybrid System was obtained from Clontech (now Takara Bio), including pGBKT7 bait vector, pGADT7 prey vector, and pGBKT7-Lamin (human lamin C amino acids 66-230), pGBKT7- p53 (mouse p53 amino acids 72-390), and pGADT7-Large-T (SV40 large-T antigen amino acids 84-708) as control plasmids. Mouse Nxf1 (C57BL/6J) open reading frame and a site-directed E610G mutation (to model CAST/Ei) were cloned into pGBKT7 after high-fidelity PCR as bait 
proteins. Mouse Nup62 open reading frame was cloned into pGADT7 as a prey for Nxf1 fusion protein. A 430-bp CUP1 promoter sequence [23] was synthesized as a gBlock Gene Fragment (IDT), with nucleotide changes to destroy the BamHI and Ndel sites, and amplified by Phusion PCR. The pGBKT7 vector was digested to remove the $705 \mathrm{bp} A D H 1$ promoter, gel purified, and used to clone the modified CUP1 promoter to create pGBKCUP1. (Figure 1A). Fluorescent proteins EGFP, mCherry, and mGrape3 [27-29] and allelic variants of Nxf1 were directionally cloned behind the CUP1 promoter in this new vector at the BamHI and Ndel sites within the multiple cloning site derived from pGBKT7. Plasmids have been deposited with Addgene, including pGBK-CUP1 empty vector (Addgene 169710) and pGBK-CUP1 with open reading frames for EGFP (170210), mCherry (170264), mGrape3 (170265), Nxf1-B (170266), and Nxf1-C (170267).

Yeast strains. Yeast transformation and growth were carried out in AH109 (MATa, trp1-901, leu2-3, 112, ura3-52, his3-200, gal4A, gal80A, LYS2 : : GAL1 UAS-GAL1 TATA- $^{-}$ HIS3, MEL1 GAL2 UAS-GAL2 TATA-ADE2, URA3::MEL1 $1_{U A S}-M E L 1_{T A T A}-l a c Z$ ), obtained as a part of the Yeast Matchmaker Two-Hybrid Systems. Transformation-competent AH109 yeast cells were prepared essentially as described [30]; briefly, cells were grown in $2 x$ YPD media (Fisher Scientific, DF0427-17-6) supplemented with $80 \mathrm{mg} / \mathrm{L}$ of adenine hemisulfate, harvested, and stored frozen at $-80^{\circ} \mathrm{C}$. AH109 was transformed with $200 \mathrm{ng}$ of plasmid for single-transformants and $200 \mathrm{ng}$ of each plasmid for co-transformants using the LiAc/SS carrier DNA/PEG method [30]. Transformed cells were grown on plates containing $1.8 \%$ agarose, minimal SD base media (Takara Bio, 630411), and a composition of every essential amino acid except tryptophan (SD/-Trp) (630413), leucine (SD/-Leu) (630414), or tryptophan and leucine (SD/-Trp/-Leu) (630417) to select for auxotrophic markers on either or both plasmids at $30^{\circ} \mathrm{C}$ for $3-5$ days. 
Western blot assay. Single yeast colonies were picked into $5 \mathrm{ml}$ of selective media for liquid culture and incubated $\sim 18-22$ hours at $30^{\circ} \mathrm{C}$ and $220 \mathrm{rpm}$ to reach saturation. Saturated cultures were diluted into fresh selective medium to $\mathrm{OD}_{600}=\sim 0.2-0.3$ in $7 \mathrm{ml}$ total and incubated $\sim 3$ hours at $30^{\circ} \mathrm{C}, 220 \mathrm{rpm}$, to obtain mid-log phase of $\mathrm{OD}_{600}=\sim 0.45$ 0.65. To induce the CUP1 promoter, $\mathrm{CuSO}_{4} \cdot 5 \mathrm{H}_{2} \mathrm{O}$ (Ricca Chemical, 2330-16) was added prior to the second incubation. After incubation, $5 \mathrm{ml}$ of chilled liquid culture was transferred to pre-chilled tubes. Cells were pelleted at $1000 \times \mathrm{g}$ and $4-10^{\circ} \mathrm{C}$ for 5 minute and washed twice with ice-cold $\mathrm{H}_{2} \mathrm{O}$. Pellets were stored at $-80^{\circ} \mathrm{C}$ until used for protein extraction. Protein was extracted essentially as described [31]; briefly, cells were resuspended in $0.2 \mathrm{M} \mathrm{NaOH}$ and incubated for 5 minutes at room temperature, pelleted, and resuspended in SDS sample buffer $(0.06 \mathrm{M}$ Tris- $\mathrm{HCl}, \mathrm{pH} 6.8,5 \%$ glycerol, $2 \%$ SDS, $4 \% \beta$-mercaptoethanol, $0.0025 \%$ bromophenol blue) supplemented with protease inhibitor (1\% Millipore Sigma P8340, 1\% PMSF), incubated at $100^{\circ} \mathrm{C}$ for 3 minutes, and centrifuged to pellet debris with the lysate retained. The volume of $\mathrm{NaOH}$ and SDS sample buffer used was dependent on the final $O D_{600}$ measurement before harvesting to account for variability in growth. Protein lysates were stored at $-20^{\circ} \mathrm{C}$ until used in western blots. Proteins ( $7 \mu$ l of extract) were separated on Laemmli SDS-PAGE gels and transferred to nitrocellulose membranes (Bio-Rad, 1620112). Membranes were stained with Ponceau-S for visualization. Epitope-tagged bait proteins were detected with mouse anti-c-myc monoclonal antibody (Invitrogen, MA1-980) and IR-680 conjugated donkey anti-mouse secondary antibody (LI-COR, 926-68072). Blots were imaged using a LI-COR Odyssey CLx imaging system. As a proxy for total protein, blots were re-probed with an anti-phosphoprotein antibody cocktail (Millipore Sigma, P3430 and P3300) and IR-800 conjugated secondary antibody. Blot images were quantified using ImageJ and data points were corrected using protein signals normalized to total protein. 
Quantitative liquid growth yeast two-hybrid assay. A single co-transformed colony ( $<2$ months old) containing both bait and prey plasmids was picked into $2-3 \mathrm{ml}$ of $\mathrm{SD} /-$ Trp/-Leu liquid media. Cultures were incubated overnight for approximately 18-22 hours at $30^{\circ} \mathrm{C}$ and $220 \mathrm{rpm}$ until saturation. The overnight cultures were diluted into fresh SD/-Trp/Leu media to a constant optical density, then split evenly into SD/-Trp/-Leu/-His media to create an $\mathrm{OD}_{600}=\sim 0.13 . \mathrm{CuSO}_{4} \cdot 5 \mathrm{H}_{2} \mathrm{O}$ was added to the yeast in SD/-Trp/-Leu/-His media to the specified concentrations. $950 \mu$ of sample was then added to a 24 -well flat bottom plate following a matrix format. The plate was incubated at $30^{\circ} \mathrm{C}$ with rocking in a chamber humidified with the same liquid media to offset evaporation. The samples were resuspended before measuring the $\mathrm{OD}_{612}$ every hour. Optical densities were measured on a Tecan i-control Infinite 200 plate reader.

\section{RESULTS}

A modified $\mathrm{Y} 2 \mathrm{H}$ bait plasmid with copper-induced expression. The classical $\mathrm{Y} 2 \mathrm{H}$

GAL4 system relies on a 700 bp $A D H 1$ promoter intended for high, constitutive expression [32]. We replaced the $A D H 1$ promoter in pGBKT7 with a modified CUP1 promoter, synthesized to destroy $\mathrm{BamHI}$ and $\mathrm{Ndel}$ restriction sites so that they remain unique in the multiple cloning site of the resulting plasmid (Figure 1A). To determine whether the modified promoter sequence retained both basal (without exogenous copper) and copperinduced activity, we cloned fluorescent protein open reading frames in-frame with the cmyc epitope tag in the vector and monitored expression of the epitope by infra-red Western blotting (Figure 1B). This showed robust and concentration-dependent induction by copper concentrations 50-200 $\mu \mathrm{M}$ (Figure 1C and S1_Table for empty vector (expressing GAL4 DNA-binding domain and c-myc epitope) and for three out of three fluorescent proteins (EGFP, mGrape3, and mCherry). To validate induction with a more challenging bait protein, we cloned full-length open reading frames for alternate alleles of 
$N x f 1$ into the new vector and compared basal and induced expression to that from the $A D H 1$ promoter of the original pGBKT7 plasmid (Figure 1D). This again showed concentration-dependent induction of bait protein expression (Figure 1E and S2_Table).

Basal (un-induced) expression from the CUP1 promoter remained detectable, but at lower levels than from the constitutive $A D H 1$ promoter, while induced expression from CUP1 achieved a higher level than $A D H 1$.

A

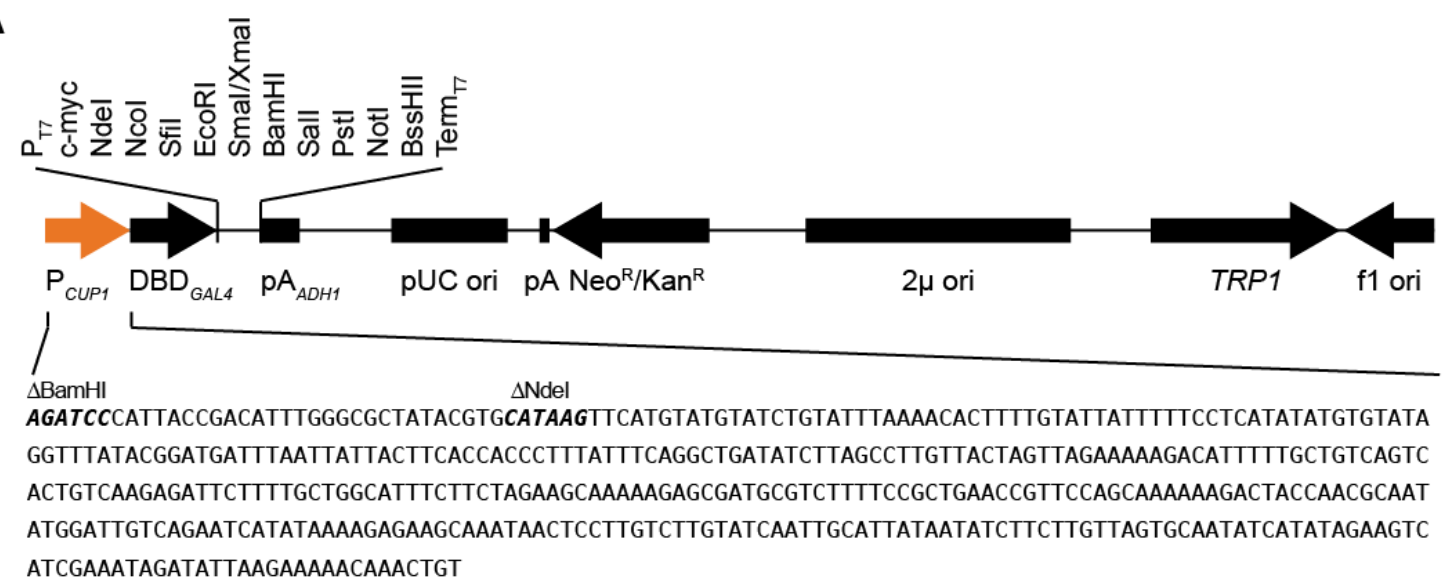

B

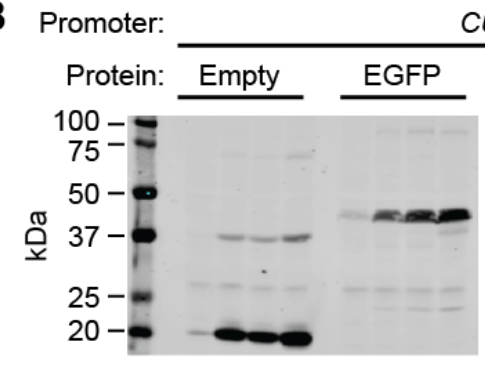

$\mathrm{CuSO}_{4}(\mu \mathrm{M}):$ 융유 융유

D Promoter: CUP1

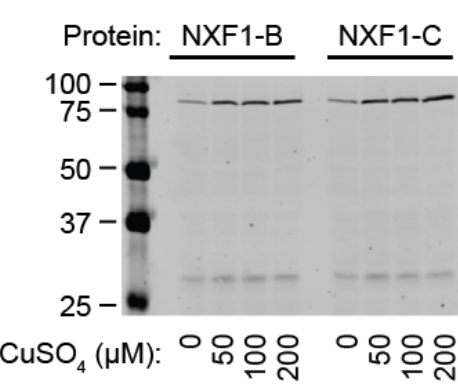

CUP1

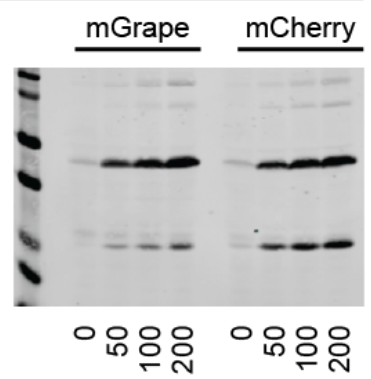

ADH1 CUP1 ADH1 CUP1

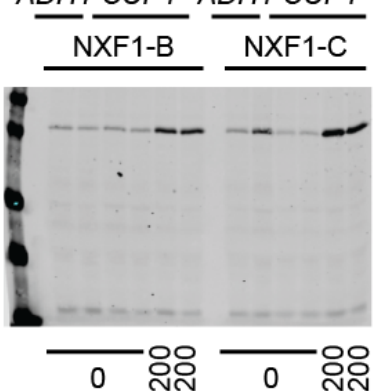

C

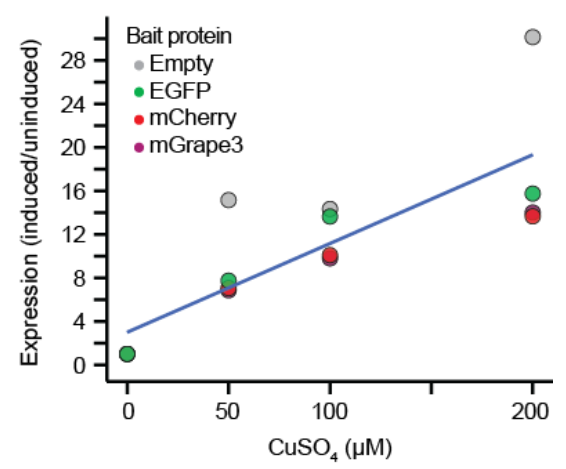

E

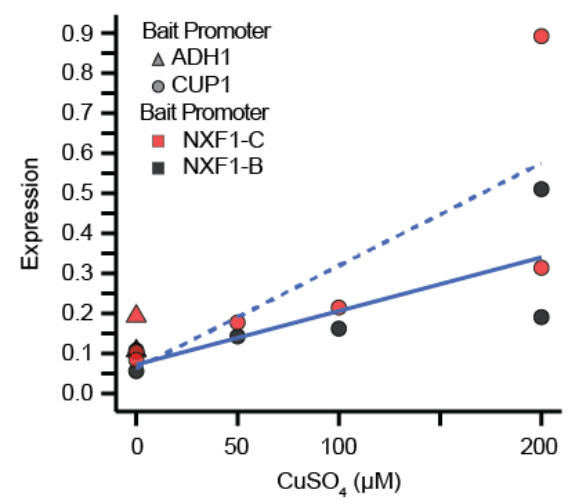


Figure 1. CUP1 promoter is dosage sensitive to copper ion concentrations. (A) Map of the 7-kb modified pGBKT7 vector with CUP1 promoter (copper arrow). Modified CUP1 promoter sequence to remove restriction sites is shown below. (B) The CUP1 promoter increased expression in response to an increase in $\mathrm{CuSO}_{4}$ concentration. Western blot data showed a change in protein expression as $\mathrm{CuSO}_{4}$ concentrations increased from 0 (uninduced) to 50,100, and $200 \mu \mathrm{M}$ (induced). Normalized expression levels were plotted relative to $0 \mathrm{mM} \mathrm{CuSO}_{4}$ (uninduced) and a best fit line was overlayed. (C) Western blot data showed greater expression when using an induced CUP1 promoter compared to the ADH1 promoter, and greater expression of NXF1-B than NXF1-C. Normalized expression levels were plotted as raw values and a best fit line was plotted only for CUP1 driven NXF1-B and NXF1-C (NXF1-B, NXF1 ${ }^{B 6}$; NXF1-C, NXF1 ${ }^{\text {CAST }}$ ).

\section{Y2H liquid growth assay validation for Nxf1:Nup62 interaction.}

To establish that copper-induced bait expression is compatible with $\mathrm{Y} 2 \mathrm{H}$ detection and relative quantification of PPI strength, we implemented a liquid growth assay (Figure 2A). We used a 24-well format with replicate cultures for allelic Nxf1 bait proteins and a known C-terminal interaction partner, Nup62 [33-40], and for positive and negative controls with plate positions alternating across replicates. We measured growth rate by optical density as a function of time while maintaining auxotrophic selection for each $\mathrm{Y} 2 \mathrm{H}$ plasmid and the integrated GAL1 $1_{U A S}-G A L 1_{T A T A}-H I S 3$ reporter in AH109, which requires PPI for growth in the absence of histidine. Growth was evident for both Nxf1 bait proteins with Nup62 prey, with faster growth for the P53 bait and SV40-LargeT prey as strong positive control and essentially zero growth among negative controls (Figure 2A and S3_Table).

We took the slope of the optical density curve during log phase as a measure to visualize growth effects of a given bait-prey pair and copper concentration (Figure 2B). For each bait-prey combination, including the $A D H 1$-driven P53 bait, increasing copper concentration decreased growth rate, consistent with its known toxicity in Saccharomyces 
$[25,26]$ (Figure 2B and S4_Table). Estimates of interaction strength in this assay will thus require controls done at the same copper concentration, at least when using growth rate as the outcome measure.

A

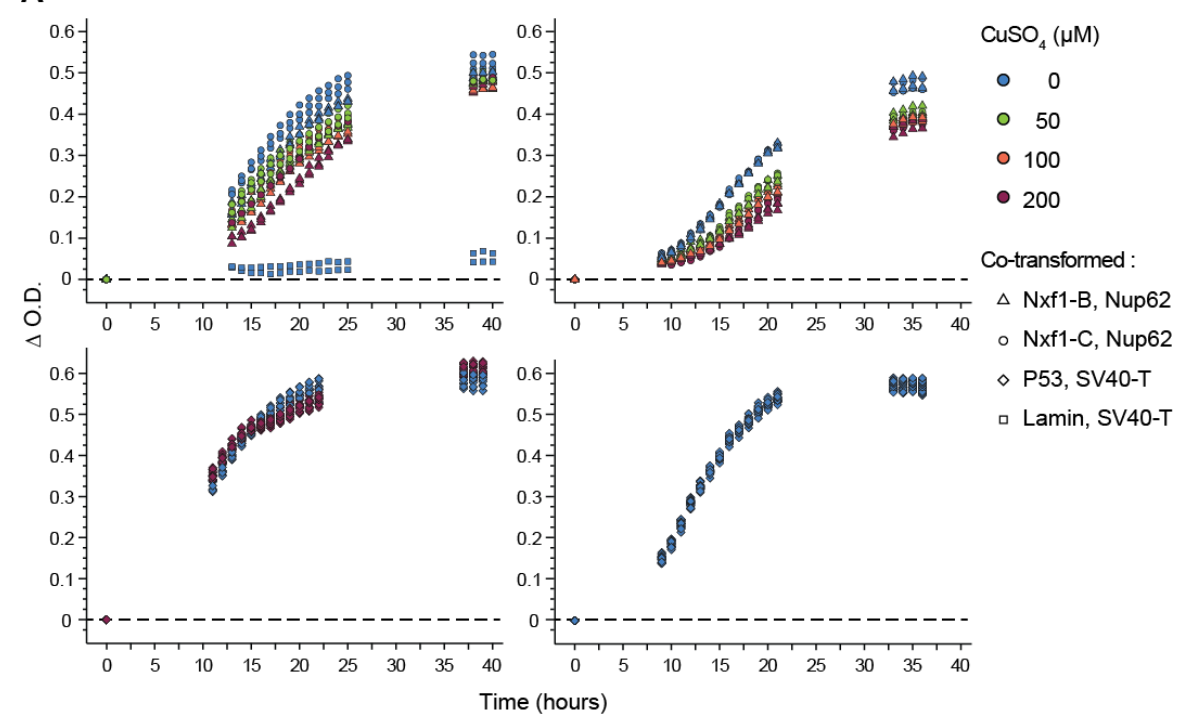

B

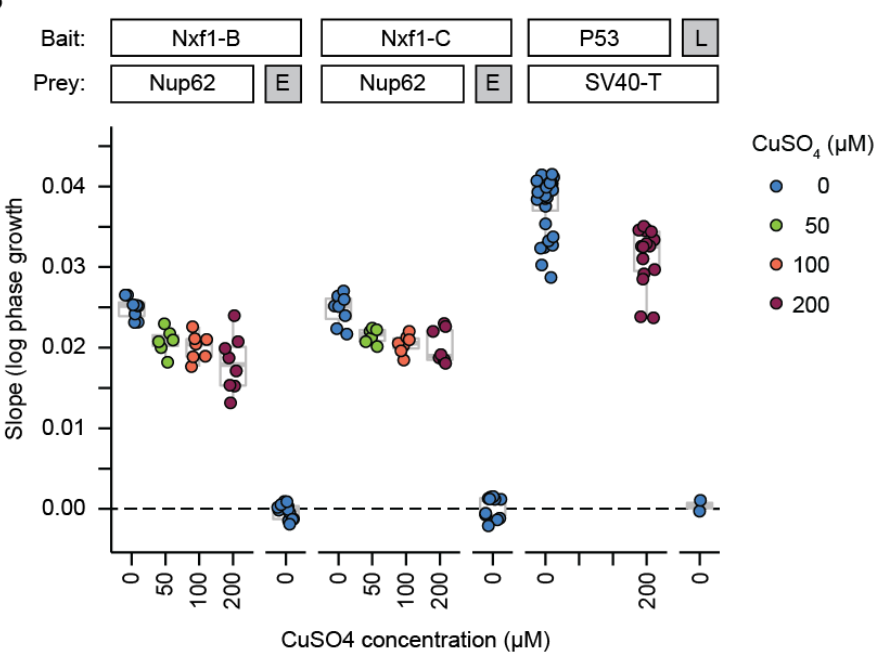

Figure 2. Y2H growth rate decreases at higher copper concentration. (A)

Representative growth curves of liquid $\mathrm{Y} 2 \mathrm{H}$ assays over time. Yeast co-transformed with either allele of Nxf1 as bait and Nup62 or empty vector prey, P53 and SV40-T (positive interaction control), or Lamin and SV4O-T (interaction negative control) were cultured in $\mathrm{SD} /$-Trp/-Leu/-His media to select for interaction in a 24 -well plate. Samples were grown 
at $30^{\circ} \mathrm{C}$ with rocking and measured at $\mathrm{OD}_{612}$ in hourly intervals up to 40 hours (NXF1-C, $N X F 1^{\text {CAST }}$; NXF1-B, $N X F 1^{B 6}$ ). $\mathrm{Y}$-axis is change in $\mathrm{OD}_{612}$ relative to time 0 . (B) Quantitative measurements of the growth rate derived from growth curves. The slope during the log linear growth phase was utilized to calculate the growth rate of each sample. Nxf1 bait expressed from with empty prey vector $(E)$ and Lamin bait $(L)$ with SV40 Large T antigen (SV40-T) were negative controls.

\section{DISCUSSION}

Yeast two-hybrid approaches have provided a flexible platform for exploring protein interactions for more than 30 years. Here, we introduced a copper-inducible bait vector based on a modified CUP1 promoter and showed $\mathrm{Y} 2 \mathrm{H}$ detection of a known interaction under both low basal expression and highly induced expression of the bait. Adding copper to liquid media allowed high level of bait protein induction and was compatible with detecting PPI, but copper toxicity decreased growth rate independent of induction, as seen by reduced growth for $A D H 1$-driven positive control pair in Figure 2. Performance of the CUP1 $\mathrm{Y} 2 \mathrm{H}$ vector might be improved in future studies by transient exposure to copper, or by use of a fluorescent or enzymatic reporter in place of growth rate as an assay. Inducible promoters from other systems or use of synthetic regulators may allow tighter regulation or induction mechanisms that are orthogonal to the biology of Saccharomyces to reduce confounding between expression level and growth. The CUP1 $\mathrm{Y} 2 \mathrm{H}$ plasmids described here have been deposited with Addgene.

Our results also showed measurable interaction between NXF1 and NUP62 independent of Nxf1 (E610G) allele. While growth rates across different copper concentrations should be interpreted with caution, $\mathrm{HIS} 3$ complementation from the $\mathrm{Y} 2 \mathrm{H}$ reporter is clear across all copper concentrations and show similar quantitative levels between Nxf1 alleles for each copper concentration tested. While quantitative growth rate for the same interaction varies across basal expression and copper concentrations for 
acute induction (or for the un-induced positive control), in each condition tested the permissive (B6) and suppressing (CAST) NXF1 proteins were not significantly different in this assay, suggesting that differential interaction with the FG-repeat containing nucleoporins may not be relevant to Nxf1-mediated genetic suppression. Future studies will be required to test whether other known NXF1-interacting proteins might show differences in interaction by the $\mathrm{Y} 2 \mathrm{H}$ assay. The approach and data here provide validation for a modified $\mathrm{Y} 2 \mathrm{H}$ bait vector to facilitate such studies.

\section{ACKNOWLEDGEMENTS}

We thank Professor Peter Novick for suggesting CUP1 as an inducible system and Professors Nathan Shaner and Roger Tsien for sharing cloned fluorescent protein genes. This work was supported by a grant from the National Institute for General Medical Sciences, R01 GM086912.

\section{REFERENCES}

1. Bachi, A., et al., The C-terminal domain of TAP interacts with the nuclear pore complex and promotes export of specific CTE-bearing RNA substrates. Rna, 2000. 6(1): p. 136-58.

2. Forler, D., et al., RanBP2/Nup358 provides a major binding site for NXF1-p15 dimers at the nuclear pore complex and functions in nuclear mRNA export. Mol Cell Biol, 2004. 24(3): p. 1155-67.

3. Gruter, P., et al., TAP, the human homolog of Mex67p, mediates CTE-dependent RNA export from the nucleus. Mol. Cell, 1998. 1(5): p. 649-59.

4. Kang, Y., H.P. Bogerd, and B.R. Cullen, Analysis of cellular factors that mediate nuclear export of RNAs bearing the Mason-Pfizer monkey virus constitutive transport element. J Virol, 2000. 74(13): p. 5863-71.

5. Kang, Y. and B.R. Cullen, The human Tap protein is a nuclear mRNA export factor that contains novel RNA-binding and nucleocytoplasmic transport sequences. Genes Dev., 1999. 13(9): p. 1126-39.

6. Katahira, J., et al., The Mex67p-mediated nuclear mRNA export pathway is conserved from yeast to human. Embo J, 1999. 18(9): p. 2593-609.

7. Segref, A., et al., Mex67p, a novel factor for nuclear mRNA export, binds to both poly $(A)+R N A$ and nuclear pores. Embo J, 1997. 16(11): p. 3256-71. 
8. Strawn, L.A., T. Shen, and S.R. Wente, The GLFG regions of Nup116p and Nup100p serve as binding sites for both Kap95p and Mex67p at the nuclear pore complex. J. Biol. Chem., 2001. 276(9): p. 6445-52.

9. Tang, H. and F. Wong-Staal, Specific interaction between RNA helicase A and Tap, two cellular proteins that bind to the constitutive transport element of type $D$ retrovirus. J Biol Chem, 2000. 275(42): p. 32694-700.

10. Wiegand, H.L., et al., Formation of Tap/NXT1 heterodimers activates Tapdependent nuclear mRNA export by enhancing recruitment to nuclear pore complexes. Mol. Cell. Biol., 2002. 22(1): p. 245-56.

11. Yoon, J.H., et al., Mex67p of Schizosaccharomyces pombe interacts with Rae1p in mediating mRNA export. Mol Cell Biol, 2000. 20(23): p. 8767-82.

12. Concepcion, D., L. Flores-Garcia, and B.A. Hamilton, Multipotent genetic suppression of retrotransposon-induced mutations by Nxf1 through fine-tuning of alternative splicing. PLoS Genet, 2009. 5(5): p. e1000484.

13. Concepcion, D., et al., Nxf1 Natural Variant E610G Is a Semi-dominant Suppressor of IAP-Induced RNA Processing Defects. PLoS Genet, 2015. 11(4): p. e1005123.

14. Floyd, J.A., et al., A natural allele of $N x f 1$ suppresses retrovirus insertional mutations. Nat Genet, 2003. 35(3): p. 221-8.

15. Fields, S. and O. Song, A novel genetic system to detect protein-protein interactions. Nature, 1989. 340(6230): p. 245-6.

16. Chien, C.T., et al., The two-hybrid system: a method to identify and clone genes for proteins that interact with a protein of interest. Proc Natl Acad Sci U S A, 1991. 88(21): p. 9578-82.

17. Young, K.H., et al., Yeast-based screening for inhibitors of RGS proteins. Methods Enzymol, 2004. 389: p. 277-301.

18. Bardou, C., et al., Peptide aptamers for small molecule drug discovery. Methods Mol Biol, 2009. 535: p. 373-88.

19. Chen, J., et al., High throughput flow cytometry based yeast two-hybrid array approach for large-scale analysis of protein-protein interactions. Cytometry A, 2012. 81(1): p. 90-8.

20. Striebinger, H., M. Koegl, and S.M. Bailer, A high-throughput yeast two-hybrid protocol to determine virus-host protein interactions. Methods Mol Biol, 2013. 1064: p. 1-15.

21. Donnard, E., et al., Yeast two-hybrid liquid screening. Methods Mol Biol, 2014. 1163: p. 97-107.

22. Butt, T.R., et al., Cloning and expression of a yeast copper metallothionein gene. Gene, 1984. 27(1): p. 23-33.

23. Butt, T.R., et al., Copper metallothionein of yeast, structure of the gene, and regulation of expression. Proc Natl Acad Sci U S A, 1984. 81(11): p. 3332-6.

24. Thiele, D.J. and D.H. Hamer, Tandemly duplicated upstream control sequences mediate copper-induced transcription of the Saccharomyces cerevisiae coppermetallothionein gene. Mol Cell Biol, 1986. 6(4): p. 1158-63. 
25. Labbé, S., Z. Zhu, and D.J. Thiele, Copper-specific transcriptional repression of yeast genes encoding critical components in the copper transport pathway. J Biol Chem, 1997. 272(25): p. 15951-8.

26. Labbé, S. and D.J. Thiele, Copper ion inducible and repressible promoter systems in yeast. Methods Enzymol, 1999. 306: p. 145-53.

27. Shaner, N.C., et al., Improved monomeric red, orange and yellow fluorescent proteins derived from Discosoma sp. red fluorescent protein. Nat Biotechnol, 2004. 22(12): p. 1567-72.

28. Shaner, N.C., P.A. Steinbach, and R.Y. Tsien, A guide to choosing fluorescent proteins. Nat Methods, 2005. 2(12): p. 905-9.

29. Lin, M.Z., et al., Autofluorescent proteins with excitation in the optical window for intravital imaging in mammals. Chem Biol, 2009. 16(11): p. 1169-79.

30. Gietz, R.D. and R.H. Schiestl, Frozen competent yeast cells that can be transformed with high efficiency using the LiAc/SS carrier DNA/PEG method. Nat Protoc, 2007. 2(1): p. 1-4.

31. Kushnirov, V.V., Rapid and reliable protein extraction from yeast. Yeast, 2000. 16(9): p. 857-60.

32. Clontech, Yeast Protocols Handbook. 2009.

33. Herold, A., et al., TAP (NXF1) belongs to a multigene family of putative RNA export factors with a conserved modular architecture. Mol Cell Biol, 2000. 20(23): p. 8996-9008.

34. Guzik, B.W., et al., NXT1 (p15) is a crucial cellular cofactor in TAP-dependent export of intron-containing RNA in mammalian cells. Mol Cell Biol, 2001. 21(7): p. 2545-54.

35. Lévesque, L., et al., RNA export mediated by tap involves NXT1-dependent interactions with the nuclear pore complex. J Biol Chem, 2001. 276(48): p. 4495362.

36. Lévesque, L., et al., Mutations in tap uncouple RNA export activity from translocation through the nuclear pore complex. Mol Biol Cell, 2006. 17(2): p. 93143.

37. $\mathrm{Yu}, \mathrm{H}$. , et al., Next-generation sequencing to generate interactome datasets. Nat Methods, 2011. 8(6): p. 478-80.

38. Rolland, T., et al., A proteome-scale map of the human interactome network. Cell, 2014. 159(5): p. 1212-1226.

39. Youn, J.Y., et al., High-Density Proximity Mapping Reveals the Subcellular Organization of mRNA-Associated Granules and Bodies. Mol Cell, 2018. 69(3): p. 517-532.e11.

40. Luck, K., et al., A reference map of the human binary protein interactome. Nature, 2020. 580(7803): p. 402-408. 